\title{
Emmanuel Bury et Carsten Meiner (sous la direction de), La Clarté à l'âge classique
}

Federico Corradi

\section{(2) OpenEdition}

1 Journals

\section{Edizione digitale}

URL: http://journals.openedition.org/studifrancesi/438

DOI: 10.4000/studifrancesi.438

ISSN: 2421-5856

\section{Editore}

Rosenberg \& Sellier

\section{Edizione cartacea}

Data di pubblicazione: 1 aprile 2015

Paginazione: 142-144

ISSN: 0039-2944

\section{Notizia bibliografica digitale}

Federico Corradi, « Emmanuel Bury et Carsten Meiner (sous la direction de), La Clarté à l'âge classique ", Studi Francesi [Online], 175 (LIX | I) | 2015, online dal 01 avril 2015, consultato il 18 septembre 2020 URL : http://journals.openedition.org/studifrancesi/438; DOI : https://doi.org/10.4000/studifrancesi. 438

Questo documento è stato generato automaticamente il 18 settembre 2020.

\section{(c) $($ ) $\odot$ EY}

Studi Francesi è distribuita con Licenza Creative Commons Attribuzione - Non commerciale - Non opere derivate 4.0 Internazionale. 


\title{
Emmanuel Bury et Carsten Meiner (sous la direction de), La Clarté à l'âge classique
}

\author{
Federico Corradi
}

\section{NOTIZIA}

EMMANUEL BURY et CARSTEN MEINER (sous la direction de), La Clarté à l'âge classique, Paris, Classiques Garnier, 2013, pp. 282.

1 Pochi concetti appaiono tanto centrali nella cultura dell'âge classique quanto quello di "clarté". Basti pensare alle due immagini che riassumono i due secoli in questione: quella del Roi-Soleil, che evidenzia l'éclat che emana dal sovrano in epoca assolutistica, e quella delle lumières, che invece disegna lo spazio, per tanti versi opposto, di un sapere che diventa critico nei confronti del potere. Ma la clarté caratterizza anche la lingua francese agli occhi dei dotti dell'epoca, motivando le affermazioni trionfalistiche sulla sua superiorità rispetto alle altre lingue moderne. E non si può dimenticare quanto essa sia centrale nel metodo cartesiano come criterio per accedere ad una conoscenza solida ed esente da errore. La parola insomma torna ossessivamente sia nel discorso filosofico che nella riflessione retorica e grammaticale, nella speculazione teologica come nella teorizzazione estetica. Ma l'unità apparente dell'oggetto di studio maschera una grande varietà di accezioni; per accorgersene basta prendere in considerazione i sinonimi possibili della parola: distinction e lumière, ma anche éclat, évidence, sublime, perspicacité, sagacité, finesse, subtilité, pénétration, ecc. Uno studio sulla clarté nell'età classica deve quindi superare le distinzioni disciplinari, abbracciare diversi campi del sapere e mostrare gli elementi di continuità ma anche le fratture che le elaborazioni ideologiche sulla clarté nascondono, deve poi elaborare delle ipotesi sulle ragioni di questa centralità. È ciò che si propongono i curatori di questo volume, che raccoglie gli atti del convegno tenutosi a Copenhagen dal 26 al 28 maggio 2005. Emmanuel Bury e Carsten Meiner sottolineano nell'introduzione come dai diversi 
contributi emergano soprattutto tre accezioni della parola: clarté come éclat, brillantezza che si impone al destinatario in modo incontestabile, clarté come evidenza oggettiva che si offre alla conoscenza, clarté come clarification, come operazione che consiste nell'illuminare, nel chiarire.

2 I primi articoli si soffermano sul versante filosofico del concetto. Jon SCHOSLER analizza uno dei problemi centrali posti dalla filosofia di Locke: conciliare le posizioni sensualiste con l'universalità dei precetti morali e con l'evidenza dei principi matematici e logici. Per Descartes la clarté (evidenza) di questi principi era basata sul loro essere idee innate ispirate da Dio. Locke, polemizzando con Descartes e con la scolastica, non rifiuta l'evidenza, ma la sua relazione necessaria con l'innatismo. L'evidenza è invece legata nel suo sistema a una semplice intuizione dell'intelletto, a una disposizione interna ad esso a cogliere in modo immediato la relazione tra due idee. I filosofi e divulgatori francesi (Condillac, Du Marsais, Quesnay, Helvétius, Fontenelle, d'Holbach, ecc.) che nel Settecento si ispirano a Locke riprendono le sue idee su questo punto, ma le loro interpretazioni spesso divergono, rivelando una comprensione solo parziale del pensiero del filosofo inglese. Denis KAMBOUCHNER si interroga sul significato del sintagma tipicamente cartesiano del "clair et distinct", che si applica alla conoscenza scientifica o a quelle conoscenze che per evidenza sono equiparabili ad essa. La concezione cartesiana della clarté e della distinction è confrontata con quella di Leibniz, che accusa Descartes di non aver sufficientemente definito questi concetti. Kambouchner conclude la sua analisi di vari passi cartesiani affermando che per Descartes «la clarté et la distinction ne se trouvent pas dans le donné perceptif lui-même, mais dans notre manière de l'appréhender» (p. 44) e invita a mettere in relazione queste caratteristiche del pensiero cartesiano con la sua scrittura, educata all'atticismo delle lettere di Guez de Balzac.

Uscendo dall'ambito filosofico, Mogens LAERKE si interroga sull'uso nelle controversie teologiche del concetto di claritas Scripturae come dispositivo critico ed ermeneutico. I protestanti, contestando la mediazione della Chiesa, sostengono che le Scritture presentano i loro significati più profondi e più sublimi in piena luce in modo che chiunque possa interpretarle nel modo giusto. Tuttavia, i due concetti opposti di claritas e di obscuritas non vanno intesi soltanto in senso statico, ma anche come categorie dinamiche: clarté equivale così a éclaircissement/clarification, obscurité a obscurcissement/ mystification. I protestanti utilizzano queste categorie per denunciare l'allontanamento dal senso letterale delle scritture, oscurato dalla ricerca dei sensi allegorici nell'esegesi scolastica. Se nella Scrittura tutto è in piena luce, la sua pretesa obscurité è dovuta all'accecamento di chi la interpreta. Ma ciò non impedisce che i protestanti sviluppino un'ermeneutica minima del testo biblico, basata sull'analogia fidei. I passi più complessi diventano chiari se spiegati confrontandoli con i passi il cui senso è più evidente. L'autore distingue poi tra le posizioni della scuola di Saumur, influenzata dal cartesianesimo, e la teologia protestante ortodossa, che hanno posizioni opposte sulla natura dell'evidenza del testo biblico.

4 Passando al versante più propriamente letterario, Volker KAPP indaga il rapporto che le riflessioni sulla poetica e la retorica tra il XVII e il xVIII secolo in Francia e in Italia stabiliscono tra la clarté, cioè la perspicuitas dei latini, e lo style moyen. Nonostante che la clarté entri sia in Italia che in Francia nella definizione dello stile medio, essa diventa, a partire da Bouhours, una discriminante per preferire questa qualità tipica della lingua francese all'affectation del Tasso e degli altri poeti italiani. Per tutto il Settecento, fino 
ad arrivare al celebre discorso di Rivarol, la clarté costituisce il principale criterio di definizione del génie della lingua francese. Fin da inizio Settecento, però, autori come Algarotti cercano di importare in Italia la clarté francese attingendo a una tradizione italiana opposta agli eccessi barocchi, quella che si rifà al Saggiatore di Galileo. Si arriva così a Parini che integra il concetto retorico della chiarezza in una riflessione estetica più ampia. Anche Anders tOFTGAARD indaga la nozione di clarté legata al "génie de la langue française", ma va a cercare le origini di questo mito nell'accusa di barbarie lanciata da Petrarca ai francesi. Ad essa si sforzano di rispondere gli umanisti d'oltralpe del XVI secolo, che lavorano sul duplice piano culturale e linguistico per far uscire la Francia e la sua lingua dall'oscurità medievale. Prendendo la parola clarté in due accezioni distinte, una più propriamente retorico-grammaticale (trasparenza, limpidezza), una politica (splendore, fama), legata all'affermazione militare della Francia, l'autore mostra che è la seconda accezione che prevale nel XVI secolo, in particolare nella Deffence di Du Bellay, ma in alcuni autori come Barthélemy Aneau e Pelletier du Mans compare anche la prima accezione: si annuncia così, seppure in maniera ancora esitante, quella celebrazione della clarté française che dominerà incontrastata da Bouhours a Rivarol.

5 Alain мÉRoт еsamina la nozione di clarté nell'ambito delle arti figurative, tracciando un parallelo tra la terminologia retorica e quella pittorica. Il suo ambito di indagine è il cosiddetto "atticismo", un movimento pittorico della metà del xvII secolo illustrato da pittori come Eustache Le Sueur e Laurent de La Hyre. In un'estetica che privilegia l' inventio e la dispositio, il disegno è fondamentale. Le singole figure e il gruppo si costruiscono poco a poco in un'unità organica attraverso una serie di aggiustamenti $\mathrm{e}$ ripensamenti che costituiscono quella che Mérot chiama una clarification. Ma la clarté propria dell'atticismo non è legata solo alla composizione che traduce in pittura l'idea, ma anche alla gradazione della luce, che sottolinea con chiarezza la disposizione rispettiva degli oggetti.

6 Giovanni BAFFETTI si propone di definire il ruolo della clarté nelle teorie dell'emblema e del motto. In entrambi i generi l'interferenza di due sistemi semiotici diversi produce effetti semantici. Se l'emblema ha un carattere più popolare e rafforza la propria immediatezza espressiva ripetendo lo stesso messaggio con strumenti diversi, il motto ha un carattere più aristocratico e rinuncia all'efficacia esplicativa del testo. Realizza così l'equilibrio, raccomandato dai teorici, tra chiarezza e oscurità, basandosi su una maggiore partecipazione del destinatario nella decifrazione del messaggio. Uno dei maggiori teorici dei due generi è Tesauro nel Cannocchiale aristotelico (1654). Tesauro descrive la chiarezza che procede dal motto come un'improvvisa illuminazione sul suo significato, che produce tanto maggiore ammirazione quanto più il significato era parso oscuro inizialmente. In De l'art des devises (1666), Le Moyne polemizza con Tesauro, sottoponendo il motto ai criteri classici della justesse, della régularité e del naturel. Alla chiarezza "che vibra come un lampo nell'intelletto" si sostituisce un'uniforme clarté che elimina ogni effetto di chiaroscuro.

7 Alain FAUdemay fa il punto sulle diverse accezioni della parola clarté nei più diversi ambiti, religioso, filosofico, linguistico, giuridico. Sottolinea in particolare l'opposizione tra una lumière che si impone in quanto éclat, che è segno del potere, come mostra l'immagine del Roi-Soleil, e una lumière che mostra, che discerne e critica trasformandosi in un contropotere, come nella metafora illuministica delle lumières. John PEDERSEN affronta invece l'evoluzione del concetto di clarté tra i due estremi 
cronologici rappresentati da Malherbe e da Diderot. I due ambiti su cui l'autore si focalizza sono l'ambito sintattico con il problema dell'ordine delle parole e l'ambito più generale della comunicazione. Per tutto il corso del Seicento il rispetto dell'ordine "naturale" delle parole è considerata la ragione dell'eccellenza della lingua francese e i principali rappresentanti del razionalismo linguistico condannano l'iperbato come infrazione a questo principio. Ma già nella Rhétorique di Bernard Lamy troviamo una posizione molto più sfumata e aperta nei confronti delle inversioni. Se la teoria dell'ordine naturale trova ancora sostenitori per tutto il Settecento, il sensualismo filosofico di un Diderot e di un Condillac portano invece ad una valorizzazione delle inversioni come espressione delle passioni. Se dall'ordine delle parole ci spostiamo sul piano della clarté delle idee, vediamo come una componente essenziale della "dottrina" di Malherbe sia il rifiuto dell'équivoque, cioè delle ambiguità di senso, un tema che sarà ripreso in senso più ampio da Boileau nella sua dodicesima satira. A questa posizione di rifiuto totale corrisponde cinquant'anni più tardi la riflessione complessa di Diderot, che nei suoi testi filosofici riflette sui limiti della comunicazione e valorizza la componente metaforica del linguaggio.

Anche Anne Elisabeth SEJTEN si concentra sulla figura di Diderot. La studiosa mostra come nel ripensamento del concetto di ragione tra Sei e Settecento sia centrale il rapporto tra filosofia e scienze della natura: se da una parte la nuova filosofia appare dipendente dalla scoperte scientifiche, dall'altra mantiene la sua autonomia e soprattutto la capacità di trarne delle conseguenze filosofiche. Diderot porta all'estremo questa tendenza a trarre dal discorso scientifico tutte le possibilità che esso offre, costruendo ardite ipotesi filosofiche tramite la congettura, la finzione e l'analogia. Il filosofo diderottiano si fa quindi interprete e non solo osservatore della natura. Da qui la tensione che attraversa l'opera di Diderot e soprattutto la Lettre sur les aveugles tra esperienza e speculazione: l'aveugle non è più tanto un soggetto clinico, quanto una figura di pensiero e un interlocutore del filosofo. Ne risulta un ripensamento del concetto di clarté che è ancora più complesso e sfumato di quanto possa sembrare ad una lettura superficiale.

9 Jean-Paul SERMAIN indaga i complessi rapporti tra Marivaux e il classicismo seicentesco. Criticato da D'Alembert per la sua oscurità, segno di un allontanamento dai grandi del secolo di Luigi XIV, Marivaux risponde definendo il pensiero come processo in cui il senso risulta dal contesto, dalle condizioni della sua produzione. Marivaux riprende il concetto barocco di ingéniosité sottomettendolo però all'esigenza classica di clarté (ingéniosité claire). L'espressione dei personaggi in se stessa è chiara e priva di ambiguità, è solo nel contesto che essa acquisisce un carattere di ingegnosità nello scarto ironico tra il discorso dell'opera e quello del personaggio, una caratteristica che Marivaux ha in comune con i grandi autori del primo Settecento. L'analisi di alcune scene de Le Jeu de l'amour et du hasard conferma che la presa in considerazione del contesto enunciativo e la complessità del dispositivo teatrale conferiscono alla clarté del discorso dei personaggi dei sensi ulteriori, in cui non è facile capire dove l'interpretazione debba fermarsi.

10 Jean-Marc CIVARDI traccia invece una breve storia del termine galimatias, uno dei tanti antonimi di clarté, nei diversi generi teatrali. Prevalente nella farsa, questo termine non designa tuttavia necessariamente l'assenza di senso, ma oscilla tra il jargon specialistico e pretenzioso dei pedanti e il linguaggio convenzionale e manierato degli amanti. Il termine ha soprattutto una valenza polemica e viene rivolto spesso da personaggi di 
buon senso a pedanti, medici e cattivi poeti. Quest'uso polemico non è frequente solo all'interno delle pièces, ma anche nei libelli critici e teorici, dove accusare di galimatias significa attribuire all'avversario infrazioni sul piano della logica, dell'espressione o della drammaturgia. L'ambito di applicazione del termine resta comunque poco preciso, e l'A. cerca di identificare alcune costanti di significato in questa varietà di usi.

11 Infine, Jean-Charles DARMON mostra il ruolo centrale svolto dall'ideale etico e retorico della clarté nella tradizione del libertinage érudit. Gassendi in particolare valorizza nell'epicureismo l'etica dello stile chiaro in funzione polemica contro i due dogmatismi, aristotelico e cartesiano. La valorizzazione della perspicuitas dello stile di Epicuro punta ad escludere ogni dissimulazione e ogni ambiguità, opponendosi in questo alla strategia libertina del chiaro-scuro. In Cyrano, invece, il discorso epicureo diventa oscuro per eccesso di éclat, cioè per l'uso e l'abuso di pointes che giocano sull'ambiguità tra l'interpretazione seria e quella ludica. Tramite l'uso sistematico del paradosso, la scrittura di Cyrano oscilla tra varie modalità conoscitive, da quella del reale o del verosimile, a quella del possibile o della pura invenzione fantastica. Ne risulta una messa in discussione della clarté come risultato dell'impostura della ragione dogmatica: a dissiparla ci pensa la retorica libertina che tende a far apparire dietro la clarté l'oscurità e la polisemia. La retorica dei libertini, insomma, associa due accezioni contrarie della clarté: da una parte il discorso libertino demistifica le superstizioni teologico-politiche, dall'altra ricorre per prudenza ad un'arte consumata della simulazione e del paradosso che copre di oscurità il pensiero. 